\title{
Review
}

Marc Bramkamp*

\section{Structure and function of bacterial dynamin-like proteins}

\begin{abstract}
Membrane dynamics are essential for numerous cellular processes in eukaryotic and prokaryotic cells. In eukaryotic cells, membrane fusion and fission are often catalyzed by large GTPases of the dynamin protein family. These proteins couple GTP hydrolysis to membrane deformation, which eventually leads to fusion or fission of the lipid bilayer. Mutations in eukaryotic dynamin-like proteins (DLPs) are associated with various diseases underscoring the importance to fully understand the biochemistry of these proteins. In recent years, a wealth of structural and biochemical data have been published that allow a detailed analysis of how dynamins or DLPs modulate biological membranes. However, less is known about the function of bacterial DLPs, although structural data exist. This review summarizes current knowledge about bacterial dynamins and discusses structural and functional properties in comparison to their eukaryotic counterparts.
\end{abstract}

Keywords: BDLP; cytokinesis; DynA; dynamin; fission; fusion; GTPase, membrane.

*Corresponding author: Marc Bramkamp, Ludwig-MaximiliansUniversity Munich, Biocenter, Department Biology I, Großhaderner Str. 2-4, D-82152 Planegg-Martinsried, Germany; and University of Cologne, Department of Chemistry, Institute of Biochemistry, Zülpicher Str. 47, D-50674 Cologne, Germany, e-mail: marc.bramkamp@Imu.de

\section{Introduction}

Biological membranes are of vital importance for cellular life. The membrane does not only separate the cytoplasm from the outside world, but is involved in subcellular compartmentalization. A hallmark of eukaryotic cells is the existence of an endomembrane system, which leads to sophisticated compartmentalization and allows creation of unique reaction compartments. Organelles such as the mitochondria, the endoplasmatic reticulum, vacuoles, or plastids are excellent examples. However, there is a constant exchange of material, including lipids, between different compartments, which require remodeling processes. Often large GTPases mediate membrane dynamic processes. The name-giving member of these proteins is the scission GTPase dynamin (Obar et al., 1990; van der Bliek and Meyerowitz, 1991). Dynamin is involved in the scission of clathrin-coated endocytotic vesicles, where it forms a ring-like collar around the neck of the budding vesicle. GTPase activity is required to release the vesicle from the plasma membrane (Praefcke and McMahon, 2004; Mettlen et al., 2009; Schmid and Frolov, 2011; Ferguson and De Camilli, 2012). Other dynamin-like proteins (DLPs) are involved in various membrane remodeling events. Scission of mitochondria is mediated by Dnm1/ Dlp (Smirnova et al., 1998; Ingerman et al., 2005), and the DLP ARC5 is involved in the division of chloroplasts (Gao et al., 2003). Other DLPs are rather involved in membrane fusion processes. Fusion of the mitochondrial outer membrane is mediated by mitofusin/fuzzy onions (Fzo) (Hales and Fuller, 1997; Hermann et al., 1998; Santel and Fuller, 2001), while fusion of the inner mitochondrial membrane is catalyzed by OPA1/Mgm1 (Alexander et al., 2000; Delettre et al., 2000; Wong et al., 2000; Meeusen et al., 2006). Recently, fusion of the endoplasmic reticulum has been associated with the DLP atlastin (Orso et al., 2009; Byrnes and Sondermann, 2011; Moss et al., 2011). Mutations in the human dynamin 2, OPA1, and atlastin lead to severe neurodegenerative disease (Charcot-Marie-Tooth disease, optic atrophy, and spastic paraplegia, respectively), and hence, these proteins are under close investigation (Knott et al., 2008). Although known for more than a decade now (van der Bliek, 1999; Leipe et al., 2002), bacterial members of the DLPs have long been neglected. The first publication about a bacterial dynamin came with the report of the structure of a DLP from the cyanobacterium Nostoc punctiforme (Low and Löwe, 2006). Cryo-electron microscopy has further shed light on the putative conformational rearrangements that allow DLPs to modulate membranes (Low et al., 2009). The first biochemical evidence that bacterial DLPs might be membrane-fusion catalysts, similar to the closely related mitofusins, was published recently (Bürmann et al., 2011). Here, the current 
understanding of bacterial DLPs in comparison to their eukaryotic counterparts is reviewed.

\section{Phylogenetic distribution of dynamin-like proteins in bacteria}

Dynamins and DLPs are ubiquitously distributed and found in animals, plants, and prokaryotes (van der Bliek, 1999). Bacterial dynamins are widely conserved. The Pfam database identifies 912 species and 1460 sequences of DLPs in bacteria (sequences in March 2012). Bacterial DLPs are found in Gram positives as well as in Gram-negative bacteria. Recently, bacterial DLPs have been identified in segmented filamentous bacteria (Pamp et al., 2012). DLPs are not only found in bacteria but are also present in a few members of the archaea (Euryarchaeota) in the class Methanomicrobia. Alignment analysis using the isolated guanine-nucleotide-binding (G-) domains reveals that bacterial DLPs fall into the mitofusin and atlastin class and are only distantly related to classical dynamins (Figures $1 \mathrm{~A}-\mathrm{B})$. This phylogenetic relation indicates a clustering of fusion GTPases. Most bacterial genomes encode at least two DLPs often organized in an operon (Bürmann et al., 2011). Sequence conservation suggests that the two genes likely originate from a gene duplication event. In firmicutes, including the pathogen Staphylococcus aureus and the various Bacillus species, the two paralogous genes have fused (Figure 2G). The DLP in Bacillus subtilis has recently been termed DynA, and the equivalents of two DLPs can easily be identified. In $B$. subtilis, they share about $16 \%$ identity and 27\% similarity (Bürmann et al., 2011). Existence of a fusion protein suggests that the bacterial DLPs work in intimate contact. As DynA is a close relative to mitofusin, it was speculated that the mitofusin/Fzo family of mitochondrial fusion proteins arose from an ancestor present in the protomitochondrion (Leipe et al., 2002).

\section{Structure of dynamin-like proteins}

Recently published structural data of full-length dynamin and various DLPs have shown that all members of the dynamin protein family share a similar architecture (Figure 2) (Prakash et al., 2000; Low and Löwe, 2006; Daumke et al., 2007; Gao et al., 2010, 2011; Faelber et al., 2011; Ford et al., 2011). Next to the globular G domain, extended $\alpha$-helical domains are evident in all DLP structures. This arrangement is a surprising theme in this class of enzymes. The classical view was that members of the dynamin family contain a middle domain (MD), a membrane-binding domain, and a GTPase effector domain (GED) (Figure 2G). However, the structures showed that MD and GED domain are part of a helical bundle and connected to the GTPase domain via a linker [neck or bundle signaling element (BSE)]. Thus, a new structure-based nomenclature is the G domain, neck/BSE, stalk/trunk, and lipid-binding domain (Figure 2). The activation of GTP hydrolysis is mediated by $\mathrm{G}$ domain dimerization, and conformational information is transferred to the trunk via the BSE (Chappie et al., 2010). The BSE is clearly distinct in the bacterial BDLP (Figure 2C) (Low and Löwe, 2006), the MxA protein (Figure 2B) (Gao et al., 2011), and the classical dynamin (Figure 2A) (Faelber et al., 2011; Ford et al., 2011). The structures of GBP1 (Figure 2D) (Prakash et al., 2000) and atlastin (Figure 2E) (Bian et al., 2011; Byrnes and Sondermann, 2011) do not show a distinct BSE. However, the stalk in these structures is also linked via a hinge to the $\mathrm{G}$ domain. Proline residues in the hinge allow domain movement of the $\mathrm{G}$ domain with respect to the stalk. The stalk (MD and GED) is contributing to dimerization and macromolecular assembly, thereby allowing cooperative GTP hydrolysis. Stalk dimerization has been shown for classical dynamin (Faelber et al., 2011; Ford et al., 2011) and the antiviral Mx proteins (Gao et al., 2010, 2011). Dimerization of atlastin requires the stalk (Bian et al., 2011; Byrnes and Sondermann, 2011; Moss et al., 2011), and the stalk region of BDLP was also suggested to be involved in oligomerization (Low and Löwe, 2006; Low et al., 2009). However, there are considerable differences in stalk oligomerization of eukaryotic and bacterial DLPs. Cryo-electron microscopy of the BDLP oligomer shows little stalk contacts (Low et al., 2009), while the stalks of dynamin (Faelber et al., 2011; Ford et al., 2011) and MxA (Gao et al., 2010, 2011) show a large dimerization surface (Figure 3B). Structural modeling of DynA predicts that D1 and D2 modules are oriented back to back, leaving the GTPase domains facing outward and enabling $\mathrm{G}$ domain dimerization in trans (Bürmann and M.B., unpublished). Upon binding to membrane lipids, it is thought that dynamin and DLPs exhibit an extended structure, which has been shown with cryo-electron microscopy (Chappie et al., 2011; Low et al., 2009). The structure of BDLP in its nucleotide-free and GDP-bound form is compactly folded with the paddle (lipid-binding motif in BDLP) in close vicinity to the GTPase domain (Figure 3A) (Low and Löwe, 2006). In contrast, the structure of nucleotide-free dynamin 1 is extended (Chappie et al., 2011; Faelber et al., 2011; Ford et al., 2011), maybe reflecting the fact that dynamin assembles readily on lipid surfaces without a nucleotide. It remains unclear whether dynamin might also adopt a closed conformation, 
A

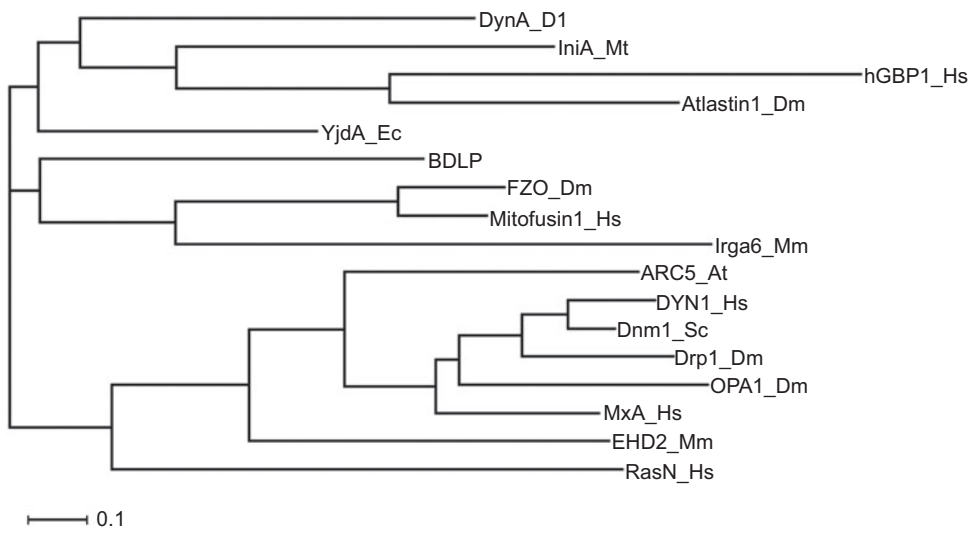

B

\begin{tabular}{|c|c|c|c|c|}
\hline & G1 (P-loop) & G2 + switch I & G3 + switch II & G4 \\
\hline Ras & LVVVGAGGVGKSALT IQLIQ & EYDPTIEDSY & I LDTAGQEEY & VGNKCDL \\
\hline Dynamin1 & IAVVGGQSAGKSSVLENFVG & SGIVTRRPLV & LVDLPGMTKV & VITKLDL \\
\hline DLP1 & IVVVGTQSSGKSSVLESLVG & TGIVTRRPLI & LVDLPGMTKV & VITKLDL \\
\hline $\mathrm{M} \times \mathbf{A}$ & IAVIGDQSSGKSSVLEALSG & SGIVTRCPLV & LIDLPGITRV & ILTKPDL \\
\hline OPA1 & VVVVGDQSAGKTSVLEMIAQ & GEMMTRSPVK & LVDLPGVINT & VLTKVDL \\
\hline Mitofusin1 & VAFFGRTSSGKSSVINAMLW & IGHITNCFLS & LVDSPGTDVT & LNNRWDA \\
\hline hGBP1 & VAIVGLYRTGKSYLMNKLAG & VQSHTKGIWM & LLDTEGLGDV & VWTLRDF \\
\hline BDLP & LLVLGDMKRGKS T FLNAL I G & VNPCTAVLTV & IVDSPGLNDT & LVNAWDQ \\
\hline DynA D1 & IAFTGHYSAGKSSLLNCLLM & SPIPTSANLV & YIDTPGIDST & IVNQIDR \\
\hline DynA D2 & LALFGGFSSGKSSFANALVG & PTPTTATINK & IVDTPGASSM & I INAADL \\
\hline Consensus & GxxxxGKS & $\mathbf{T}$ & $\mathrm{D} \mathbf{x} \mathbf{x} G$ & $\begin{array}{l}N K \mathbf{x} D \\
T R \mathbf{x} D\end{array}$ \\
\hline
\end{tabular}

Figure 1 Phylogeny of dynamin family members.

(A) Phylogenetic analysis of various dynamin family members. The alignment was made using ClustalOmega (Sievers et al., 2011), and the neighbor-joining method was used for tree calculation. The sequences of $\mathrm{G}$ domains ranging from $\mathrm{G} 1$ to the $\mathrm{G} 4$ motif of the proteins were submitted. The phylogram shows that bacterial dynamins (BDLP, DynA, IniA, and YjdA) are related to mitofusins, atlastins, and GBPs, likely suggesting a clustering of GTPases involved in membrane fusion. Fission GTPase such as dynamin (DYN1, Dnm1, Drp1) also cluster together. Interestingly, the inner mitochondrial fusion GTPase OPA1 clusters together with the fission GTPases. At, Arabidopsis thaliana; Bs, Bacillus subtilis; Dm, Drosophila melanogaster; Ec Escherichia coli; Hs, Homo sapiens; Mm, Mus musculus; Mt, Mycobacterium tuberculosis, NP, Nostoc punctiforme, Sc, Saccharomyces cerevisiae. (B) Alignment of GTPase domains showing the conserved four GTPase motifs. Critical residues are highlighted in blue. For the $B$. subtilis protein fusion, DLP DynA sequences of the D1 and D2 modules are shown.

for example, in its tetrameric form. An interesting difference between bacterial and eukaryotic dynamins/DLPs is the apparent difference in macromolecular assembly (Figure 3). G domain dimerization in the MxA and dynamin oligomer occurs via $\mathrm{G}$ domains of neighboring rungs on the helix/rings (Chappie et al., 2011; Faelber et al., 2011; Ford et al., 2011; Gao et al., 2011). In contrast, cryo-electron microscopy data of the BDLP oligomer shows G domain dimerization between neighboring monomers on the same rung (Figure 3) (Low et al., 2009).

\section{GTP hydrolysis and G domain dimerization interface}

A hallmark of dynamin and DLPs is their ability to bind and hydrolyze GTP. The nucleotide-binding domain is the most conserved part of the otherwise modular architecture of dynamins (Figures 1B and 2). In contrast to small signaling GTPases, DLPs are large GTPases of around 70-100 kDa. Dynamins and the bacterial DLPs belong to the translation factor-related (Trafac) GTPases (Leipe et al., 2002). The nucleotide-binding domains (G domain) in DLPs contain four well-characterized motifs (G1-4) (Figure 1B) and a less well-conserved G5 motif. These include the highly conserved P-loop or G1 motif (GxxxxGK[ST]), the G2 or switch I (a highly conserved threonine residue), and the G3 or switch II (DxxG). GTPutilizing enzymes share the signature G4 motif [NT]KxD (Leipe et al., 2002). Additional contacts to the nucleotide are made by residues similar to the G5 motif (SA[KL]) of small GTPases. In the Dictyostelium discoideum dynamin A, these contacts are mediated by asparagine (238) and arginine (239) residues (Niemann et al., 2001). A unifying characteristic in eukaryotic and prokaryotic DLPs is 
A

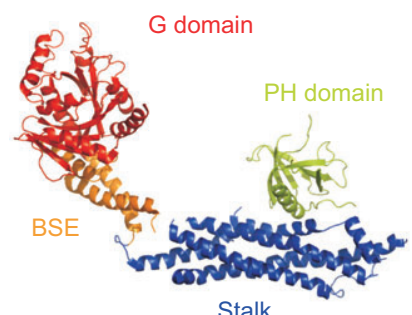

Dynamin 1 (apo)

C

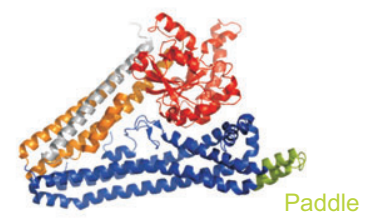

BDLP (GDP)

E

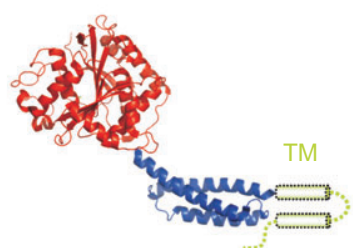

Atlastin (GDP)
B

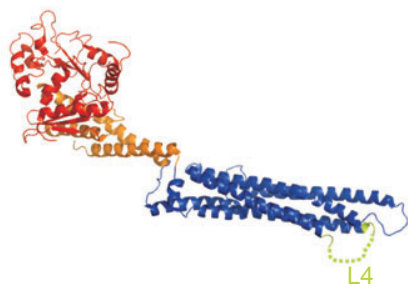

MxA (apo)

D

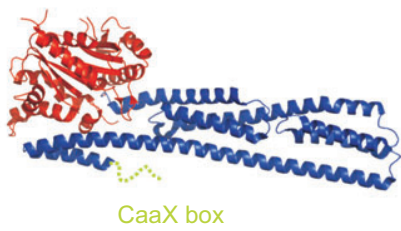

GBP1 (apo)

F

EH domain

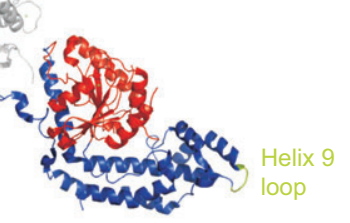

EHD2 (ANP)
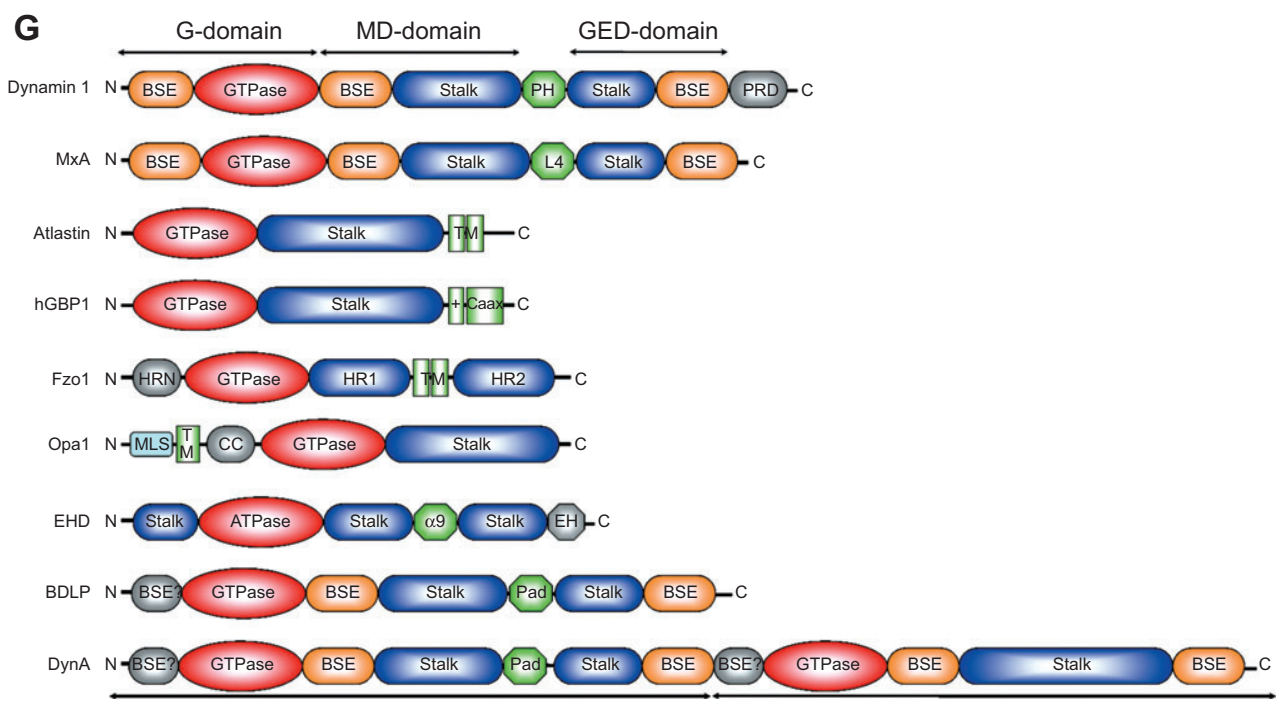

D1

D2

Figure 2 Modular architecture of dynamin family members.

Members of the dynamin family of proteins have a modular domain arrangement $(A-F)$. Molecule names and nucleotide state are indicated below the structures. Structures of dynamin (A) and DLPs (B-F) show conserved structural features. Dynamin ( $A, p d b$ code $3 S N H)$, MxA (B, 3SZR), BDLP (C, 2J68), GBP1 (D, 1DG3), atlastin (E, 3Q5D), EHD2 (F, 2QPT). Common to all is the nucleotide-binding domain (G domain, red), a helical stalk domain (blue), and a lipid-binding module (green). Phospholipid binding is mediated by different structural elements located between stalk helices. Classical dynamins use a pleckstrin homology domain (PH) for membrane binding. Other DLPs have either transmembrane helices (TM) or exposed loops (L4, helix 9), isoprenylation sites (CaaX), and paddle domains (Pad). A cartoon summarizes the modular domain arrangement $(G)$. The classical domain nomenclature is shown above (G, MD, and GED domain). Based on the structural data dynamin (A), MxA (B) and BDLP (C) show a clear bundle signaling element (BSE, orange). The bacterial DLP DynA, found in firmicutes, is a remarkable fusion of two DLPs, where only the first DLP domain (D1) harbors a membrane-binding paddle domain. The mitochondrial fusion GTPase Fzo1 has three coiled-coil motifs (HRN, HR1-2). These coiled coils are likely similar to the stalk domains of other DLPs. Opa1 contains an N-terminal mitochondria localization signal (MLS) followed by a transmembrane helix. The coiled-coil motif preceding the $\mathrm{G}$ domain (CC) of Opa1, as well as the HRN in Fzo1, might indeed serve as BSEs. 

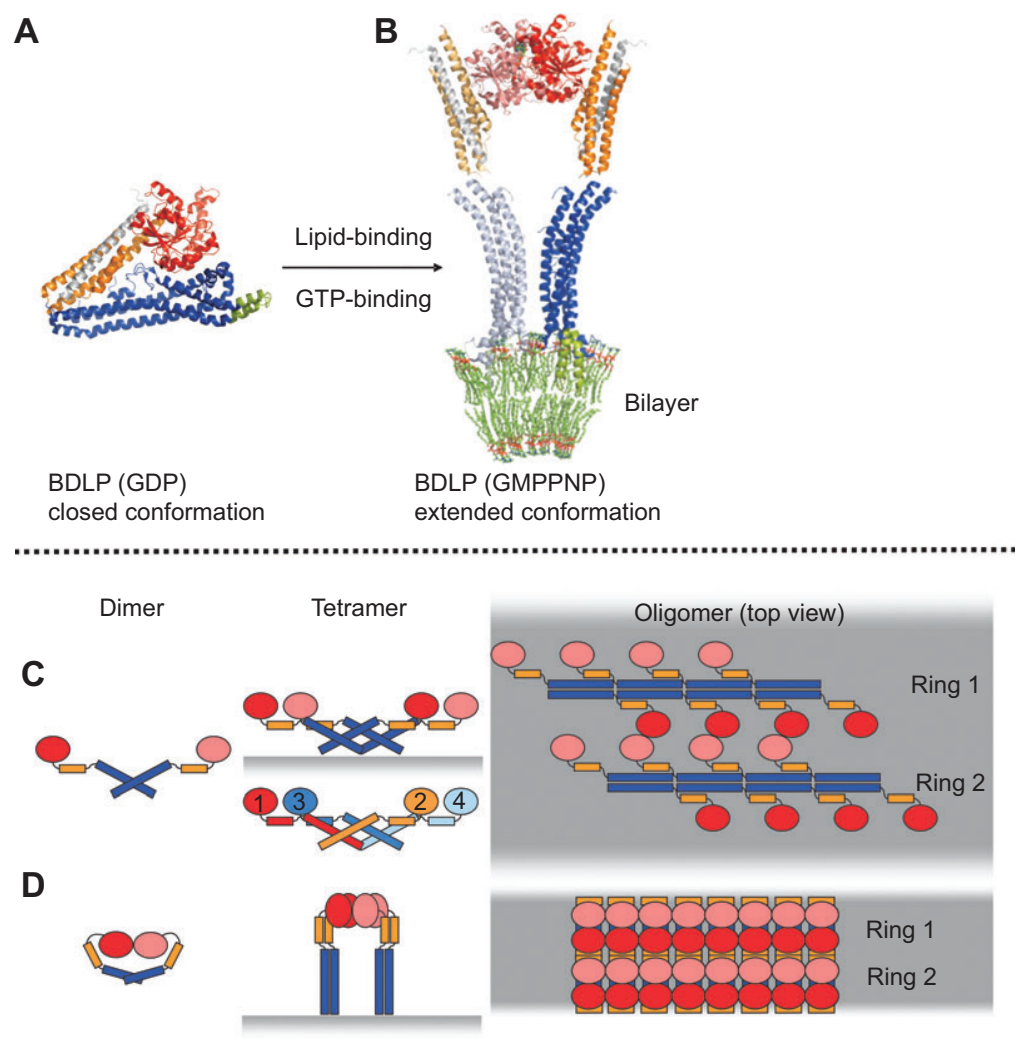

Figure 3 Oligomerization and assembly of MxA and BDLP.

(A, B) The structures of N. punctiforme BDLP (Low and Löwe, 2006; Low et al., 2009) show a dramatic domain movement upon binding of GTP and lipids. The BDLP structure is closed in its GDP-bound (and in its apo-) form (A, 1J68). However, molecular modeling of the structure into cryo-electron microscopy reconstructions revealed an extended molecule showing the classical $G$ domain dimerization contacts (B, 2W6D). The paddle domain is inserted into the outer leaflet of the lipid bilayer and causes membrane curvature. Although $\mathrm{G}$ domain dimerization is conserved between bacterial and eukaryotic DLPs and dynamin, there seems to be a significant difference in the assembly of the higher oligomers. (C, D) A cartoon illustrates the putative dimer, tetramer and oligomer assemblies of MxA (C) and BDLP (D). The macromolecular assembly of dynamin would largely resemble the MxA oligomer, with the exception that dynamin oligomers form a helical arrangement. Dimer interfaces in the stalk and $\mathrm{G}$ domain are similar in MxA and dynamin. MxA assembles in ring fashion with criss-cross contacts of their stalk domains that run in an angle of around $45^{\circ}$ across the lipid surface. (C). In striking contrast, bacterial BDLP binds to the membrane surface (gray) with an orthogonal assembly of the stalk domains. Protein contacts are via lateral contacts of the $\mathrm{H} 4 \mathrm{helix}$ at the site of the G domains and also via neighboring BSE domains. G domains are in red, stalk domains are in blue and BSE domains are in orange.

the dimerization interface of the $G$ domains. Dynamin and DLPs were therefore classified as G proteins activated by nucleotide-dependent dimerizations (GAD) (Gasper et al., 2009). This dimerization has been shown for the bacterial BDLP (Low and Löwe, 2006; Low et al., 2009), dynamin 1 (Chappie et al., 2010, 2011), GBP1 (Ghosh et al., 2006), atlastin (Bian et al., 2011; Byrnes and Sondermann, 2011), and EHD2 (Daumke et al., 2007). Even the more distantly related GTPases such as septins display a similar G domain dimerization (reviewed in Gasper et al., 2009). The $G$ domain dimer interface is formed upon GTPbinding and remains after hydrolysis in the GDP state [for review, see Low and Löwe (2010)]. Binding of GDP to BDLP promotes formation of a crystallographic dimer. Here, the nucleotides are positioned between the adjacent $\mathrm{G}$ domains, and glutamate 245 (helix 9) might contribute to the stabilization of the nucleotide bound to the opposing $\mathrm{G}$ domain (Low and Löwe, 2006). In the GDP bound state switch II including the conserved aspartate (D98 in BDLP) is largely disordered, allowing access to the active site, which is occluded by Asp98 in the nucleotide free form (Low and Löwe, 2006). The G domain dimers of GBP1 and dynamin 1 show similar dimerization interfaces (Ghosh et al., 2006; Chappie et al., 2010). The crystal structure of the dynamin $1 \mathrm{G}$ domain (including the GTPase effector domain), in the presence of the transition state mimic GDP. $\mathrm{AlF}_{4}^{-}$, reveals the coordination of the nucleotides in cis and trans by the G4 motif. Aspartate D211 stabilizes the nucleotides in trans by forming hydrogen bonds with the guanine base, which is also the case in BDLP (Chappie et al., 2010). 
Activation of GTPase activity requires structural rearrangements that allow the nucleophilic attack on the $y$-phosphate, charge compensation, and stabilization of the switch regions (Chappie et al., 2010). For dynamin, it has been convincingly shown that cis and trans interactions occur that stabilize the P-loop and switch 1 conformation. Charge compensation in the dynamin $\mathrm{G}$ domain may be accomplished by a sodium ion. This is in contrast to the case in GBP1, where an arginine residue from the P-loop flips toward the phosphates of the nucleotide in cis, enabling efficient nucleotide hydrolysis (Ghosh et al., 2006). In BDLP, Lys 79 is thought to function in a similar way (Low and Löwe, 2006). The conserved P-loop lysine (K 44 in dynamin 1) coordinates with the $\beta$ - and $\gamma$-phosphates of the nucleotide and contributes to charge compensation. Mutation of the P-loop lysine leads to loss of GTP binding and hydrolysis in dynamin 1 (Damke et al., 2001), as well as in the bacterial DynA and BDLP (Low and Löwe, 2006; Bürmann et al., 2011). Similar mutations have been made for GBP1 (Praefcke et al., 2004), Fzo1 (Hermann et al., 1998), and Dlp (Zhang et al., 2011). GTPase activation for dynamin is established in cis. Mutations in the P-loop affect stimulated GTPase activity, while self-assembly is not affected (Chappie et al., 2010). Activation of GTP hydrolysis in the B. subtilis DynA requires GTP binding to both $\mathrm{G}$ domains of the full-length protein (Bürmann et al., 2011).

Dynamins have been termed mechanochemical enzymes as they couple GTP hydrolysis to mechanical work at the membrane (Warnock and Schmid, 1996). Although, it is still debated where exactly GTP hydrolysis is essential in the reaction process, it is clear that a cooperative mechanism must work in all family members (a notable exception is the isolated BDLP as described below). All dynamin protein family members have a low affinity for GTP and exhibit a high basal GTPase activity (Praefcke and McMahon, 2004; Schmid and Frolov, 2011). GTPase activity of dynamin is stimulated in the presence of liposomes (Warnock and Schmid, 1996). In contrast, in bacterial DLPs tested so far, BDLP and DynA show no liposome-stimulated GTPase activity. Addition of liposomes to DynA has only a marginal effect on activity, which is likely due to the local enrichment of the protein on the liposome surface. Maximal turnover rates for DLPs range from 26/ min to 190/min (Praefcke and McMahon, 2004) to as low as 5.6/h for EHD2 (Daumke et al., 2007). The maximum turnover rate of $B$. subtilis DynA is around $28 / \mathrm{min}$ at 1.5 $\mu \mathrm{M}$ protein concentration. This is a sixfold increase in specific activity compared to that at $0.25 \mu \mathrm{M}$ DynA ( 4.5/ min). In common, however, all eukaryotic dynamins show a clear cooperative GTP hydrolysis. The situation in the bacterial DLPs is not clear with DynA behaving cooperatively, leaving BDLP as the exception to the rule. Instead, a Michaelis-Menten kinetic of BDLP $\left(K_{\mathrm{m}} \sim 69 \mu \mathrm{M}, k_{\text {cat }}\right.$ $\sim 0.53 / \mathrm{min}$ ) was stated (Low and Löwe, 2006). However, here, only isolated BDLP1 paralog was used, while DynA is a fusion product encompassing the homologs of BDLP1 and BDLP2 from N. punctiforme. Also, mixing recombinant D1 and D2 modules of DynA did not restore GTP hydrolysis, indicating that intimate contact between the two domains is essential for correct functioning (Bürmann et al., 2011), maybe due to a functional interaction of D1 BSE/GED with the D2 G domain. Interestingly, the quaternary structure of the unassembled dynamin 1 is a tetramer (Schmid and Frolov, 2011), which would be similar to the observed DynA dimer in terms of GTPase domains. GTP binding to both GTPase domains is essential for hydrolysis in DynA. It has been shown that the $\mathrm{D} 1$ and the $\mathrm{D} 2$ modules of DynA undergo homotypic interactions (Bürmann et al., 2011), likely reflecting the nucleotide-dependent dimerization, which is common to dynamin and DLPs.

\section{Lipid-binding mechanisms}

Classical dynamins harbor a pleckstrin homology $(\mathrm{PH})$ domain for membrane interaction. The $\mathrm{PH}$ domain binds to phosphoinositides (phosphatidylinositol (4,5)-bisphosphate $(\mathrm{PI}(4,5) \mathrm{P} 2)$ and phosphatidylinositol $(3,4,5)$-trisphosphate $(\mathrm{PI}(3,4,5) \mathrm{P} 3)$. This allows directed binding to membrane areas enriched in PIP (Downing et al., 1994; Lemmon, 2007; Ramachandran et al., 2009; Ford et al., 2011). The PH domain is embedded in the stalk domain (Figure 2). Other dynamin-like proteins have evolved different membrane-binding mechanisms (Figure 2). Mitofusins, OPA1 (Mgm1), and atlastins have transmembrane helices that anchor the proteins permanently to the target membranes. In contrast, interferon-induced human guanylate-binding protein GBP1 has a CaaX box motif (Schwemmle and Staeheli, 1994; Fres et al., 2010), where isoprenoids are postranslationally linked to the invariant cysteine. The CaaX box in hGBP1 is preceded by a polybasic motif that supports binding to negatively charged phospholipids (Fres et al., 2010); however, not all GBPs contain a CaaX box motif. Lipid-binding mechanisms in these proteins remain unclear. The antiviral Mx proteins bind via an exposed loop (Loop 4) to membranes (von der Malsburg et al., 2011). Distantly related EHD ATPases also expose a lipid-binding loop (Daumke et al., 2007). Bacterial dynamins share a similar membrane-binding mechanism. The structure of BDLP revealed a paddle domain 
that is inserted into the lipid bilayer (Low and Löwe, 2006; Low et al., 2009). Similar to other DLPs and dynamin, the paddle is embedded in the stalk domain and exposes a lipid-binding loop. Mutational analysis has shown that two leucine residues (L576 and L577) as well as phenylalanine F583 in the paddle region contribute to lipid-binding (Low et al., 2009). Thus, all known members of the dynamin family share a modular design, with an exposed lipid-binding module at the tip of an extended $\alpha$-helical stalk domain (Figure 2).

\section{Mechanisms of membrane fusion by bacterial DLPs}

Dynamins and DLPs are involved in various membrane dynamics, such as fusion and scission of membranes. Dependent on their role, the proteins have evolved specific characteristics. Fusion processes have in common that membranes are tethered in trans, before fusion occurs. This has been nicely shown for fusion of the outer and inner mitochondrial membrane (Westermann, 2010). Recently, atlastins have been shown to mediate membrane fusion of ER tubes (Orso et al., 2009; Moss et al., 2011). Strikingly, here, a three-way junction is established. Structures of atlastin show a $90^{\circ}$ rotation of the GTPase domain with respect to the stalk (Bian et al., 2011; Byrnes and Sondermann, 2011). A similar rotation has been proposed for the bacterial BDLP (Low et al., 2009). Upon lipid-binding, BDLP adopts an extended conformation seen as radial spikes decorating the lipid tube in cryo-EM images (Figure 3) (Low et al., 2009). The B. subtilis DLP DynA was shown to tether membranes in trans in a cellular environment as well as in vitro (Bürmann et al., 2011).

Strikingly, only the DynA D1 module confers lipid-binding (Bürmann et al., 2011). Structural modeling of DynA reveals that likely only the $\mathrm{D} 1$ module has a paddle region similar to BDLP, while the D2 module lacks this region (Figure 2G). The biochemical data thus support well the structural model. The isolated D1 module alone binds with high affinity to membranes, similar to full-length DynA. Binding is not influenced by guanosine nucleotides (Bürmann et al., 2011). Recent experiments have revealed that DynA binds to negatively charged phospholipids such as cardiolipin and phosphatidylglycerol at physiological salt concentrations (P. Sawant and M. B., unpublished). Transmission EM clearly revealed an ordered self-assembly of DynA on liposome surfaces in the absence of a nucleotide, leading to a deformation of the vesicle (Bürmann et al., 2011). Thus, induction of curvature is not a result of GTP hydrolysis, but merely a result of organized assembly into a macromolecular complex. In vivo visualization of DynA in B. subtilis using GFP fusion constructs shows membrane association of the protein, and often, focal localization can be observed (Bürmann et al., 2011). However, this focal localization of DynA is not an innate property of the proteins as expression of DynA-GFP in baker's yeast never revealed focal localization. Of note, expression of DynA in yeast leads to a striking tethering of vacuolar membranes to the cytoplasmic membrane, indicating the ability of DynA to tether membranes in trans in a cellular environment. FRAP experiments showed that DynA-GFP was entirely mobile when assayed in yeast $(\tau \sim 5 \mathrm{~s})$. In contrast FRAP experiments in $B$. subtilis revealed that only one-third of the protein is mobile, and half-maximal recovery was reached after 17 s (M.B. and Nina Ebert, unpublished). Interestingly, a GTP-binding mutant (K56A/K625A) has a half-maximal recovery close to the values detected in yeast ( $\tau \sim 5 \mathrm{~s}$ ). A plausible explanation is that mobility of DynA and focal concentration of the protein in bacterial cells depends on a trans-acting factor, likely a lipid or a protein partner. Further, these results show that GTP-binding (and likely hydrolysis) do affect the mobility on the membrane. It might be speculated that GTP binding/hydrolysis is needed for the release of bacterial dynamins from the membrane. This idea is supported by a structural analysis of lipid-bound BDLP. BDLP does not bind efficiently to liposomes in its GDP-bound state, suggesting that hydrolysis and, in turn, the subsequent conformational change may help to release the protein from the membrane (Low et al., 2009). However, it seems essential at least for BDLP to bind GTP in order to open the closed conformation and allow lipid binding (Figure 3A). In contrast to this assumption, DynA binds perfectly well to lipid in the absence of any nucleotide (Bürmann et al., 2011). This obvious difference between BDLP and DynA may result from DynA being a fusion of two DLPs, likely in back-to-back orientation. Thus, DynA might show different conformational dynamics compared to BDLP, leaving the enzyme in a structurally more expanded conformation even in its apo form. GTP hydrolysis as part of the membrane-binding cycle has also been proposed for dynamin (Bashkirov et al., 2008; Pucadyil and Schmid, 2008). GTP addition to membrane-assembled dynamin triggers disassembly of the oligomer (Pucadyil and Schmid, 2008). Thus, GTP hydrolysis is not coupled to membrane deformation per se, but rather is needed for the correct cycling between membrane-bound and soluble form. A passive model of membrane deformation has also been proposed for BDLP (Low et al., 2009). In summary, oligomerization of the DLPs on the target membrane provides enough binding energy to deform the membrane. 
As described above, DynA is able to tether membranes in trans. In vitro, mixing of DynA with preformed liposomes shows quick aggregation of liposomes (Bürmann et al., 2011). This aggregation critically depends on the D1 module, while the isolated D2 module is not able to support membrane tethering. Using isolated DynA-GFP, the aggregation process could be visualized and suggests a cooperative mechanism for membrane tethering in trans. When lipid-bound DynA was proteolytically removed from the aggregates in vitro, large unilamellar fusion products were observed (Bürmann et al., 2011). Importantly, fusion was nucleotide independent, only requiring magnesium ions. Again, this would argue in favor of nucleotide hydrolysis as being important for protein release from the membrane. Tethering as an important prerequisite for fusion has been described for Mgm1 (OPA1). Mgm1 functions as a large and a small isoform in fusion of the inner mitochondrial membrane. Both isoforms are inactive on their own, but together, they form a functional dimer in trans that serves as nucleation point for the generation of a higherorder scaffold (DeVay et al., 2009). Interestingly, dimer formation is cardiolipin dependent, and hence, similarities to DynA are evident. The formation of trans tethering complexes has partially been solved for mitofusins. Here, a heptad repeat region in helix HR2 is discussed to assist in the dimerization of mitofusins in trans by establishing an antiparallel coiled coil (Koshiba et al., 2004). However, these data are controversial (Low and Löwe, 2009). Likely, the tethering mechanism is more complex. The yeast mitofusin Fzo1 shows G domain-dependent homodimerization. Upon further oligomerization, the outer membranes of the mitochondria are tied together, allowing membrane fusion (Anton et al., 2011; Cohen et al., 2011). Interestingly, Fzo is ubiquitinated by Mdm30, priming Fzo for degradation. The degradation is an essential step to achieve full fusion, likely by removing the DLP scaffold and leaving the membrane in an energetically unfavorable, highly bent state that relaxes upon fusion (Anton et al., 2011; Cohen et al., 2011). This is an interesting parallel to the observations reported for bacterial DynA. Visualization of large liposomes was only possible after removal of the DynA scaffold with proteases in vitro (Bürmann et al., 2011). How bacterial DLPs mediate trans tethering remains elusive. Possibly, the formation of $\mathrm{G}$ domain dimers of opposing DynA molecules is sufficient, but interaction of the stalk domains may also be involved.

Fusion DLPs interact with membrane proteins that help to assemble the DLP scaffold. Fzo1 interacts with Ugo1 in the mitochondrial outer membrane (Sesaki and Jensen, 2001, 2004). Atlastins interact with the reticulons and DP1 proteins in the ER membrane (Shibata et al., 2008; Hu et al., 2008, 2009). Recently, several membrane integral proteins have been identified that interact with DynA. The cell division proteins MinJ (Bramkamp et al., 2008; Bürmann et al., 2011) were shown to be essential for correct localization of DynA, and a bacterial two-hybrid screen identified the uncharacterized membrane protein YneK as the interaction partner of DynA (Bürmann et al., 2012, see below). Thus, one might speculate about a common mechanism for targeting fusion DLPs to the correct sites on the membranes. Further, the membrane interaction partners may serve as nucleation points to start oligomerization.

A major challenge during the process of membrane scission and fusion is maintenance of the integrity of the membrane throughout the process in order to avoid content leakage. Although fusion and fission of biological membranes are opposing events, both processes likely have a hemifusion state as intermediate. Fusion of just one leaflet of the lipid bilayer prevents leakage of the soluble components encapsulated in the membrane compartment. The fact that the hemifusion state is a common intermediate for fusion and fission events explains how members of the dynamin protein family can catalyze fusion and fission (Chernomordik and Kozlov, 2003; Kozlovsky and Kozlov, 2003). Thus, a central step in the reaction cycle is the coordinated assembly of the protein at the membrane, thereby imposing curvature on the membrane. The highly curved membrane enters a fusogenic state in which, first, a hemifusion stalk/interface is produced (Kozlovsky and Kozlov, 2003; Pawlowski, 2010). Using a FRET-based lipidmixing assay, it could be shown that DynA is at least able to promote hemifusion. However, visualization of large unilamellar vesicles suggests that also content mixing occurs after DynA-mediated membrane fusion (Bürmann et al., 2011).

\section{Cellular role of bacterial dynamin- like proteins}

Although structural data and biochemical analyses of bacterial DLPs are available, a clear-cut cellular role remains somewhat elusive. Eukaryotic dynamin is involved in the release of clathrin-coated vesicles, but so far, no similar process has been observed in bacteria. Bacteria do release membrane vesicles to the environment (Deatherage et al., 2009; Deatherage and Cookson, 2012). However, if a dynamin-like function of bacterial DLPs would be predicted, their localization must be extracellular (where nucleotides are unavailable) rather than cytoplasmatic. 


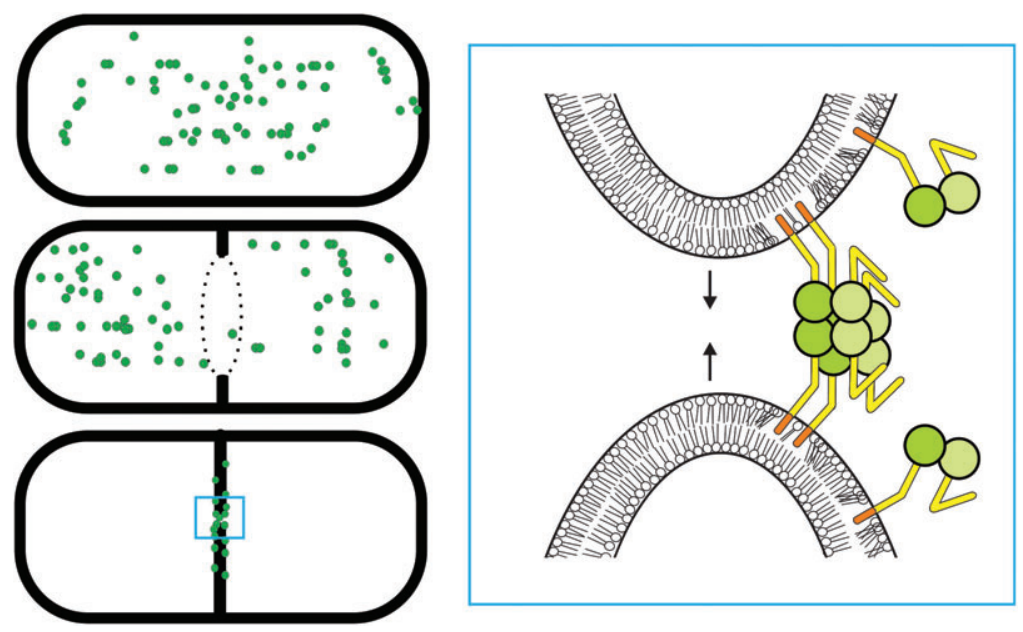

Figure 4 Putative function of Bacillus DynA during cytokinesis.

The $B$. subtilis protein DynA is localized in the cytoplasm, but can bind to negatively charged phospholipids. Although, a clear phenotype of $d y n A$ mutants has not been described, septal defects during osmotic shock and binding to cytokinetic proteins such as MinJ, suggest that DynA might participate in cytokinesis. A plausible function would be induction of membrane curvature to the inward-growing septal membranes, bringing them into a fusogenic state. Induction of curvature is achieved by insertion of the paddle domain (orange) of the D1 module (dark green). Homotypic contacts between D1 and D2 (light green) allow membrane tethering in trans, finally leading to membrane fusion. Cooperative GTP hydrolysis likely releases the protein from the membrane.

So far, there is no evidence for any export of bacterial DLPs. Another scenario would be a role similar to Mx proteins in eukaryotes, which act as antiviral proteins. However, influence of bacterial DLPs on phage infection has not been described yet. Localization of BDLP in N. punctiforme and DynA in B. subtilis reveals a septal enrichment (Low and Löwe, 2006; Bürmann et al., 2011) of an otherwise uniform or punctuate pattern. This might be in line with a role of bacterial dynamins in cytokinesis. Indeed, it could be shown that localization of DynA is grossly altered in the absence of MinJ, a cytokinetic protein in B. subtilis (Bramkamp et al., 2008; Patrick and Kearns, 2008; Bürmann et al., 2011). A dynA null mutation in B. subtilis has, however, no effect on vegetative growth. Only under osmotic shock conditions did transmission EM reveal deformed septa in cells lacking DynA (S. van Baarle, F. Bürmann and M.B., unpublished). Thus, it could be speculated whether DynA helps in catalyzing membrane fusion at the end of cytokinesis at least under osmotic stress conditions. In line with this idea, it seems plausible that many bacterial species encode bacterial dynamins, as osmotic stress is one of the most often encountered stresses for bacteria, and many emergency systems have evolved to counteract osmotic stress (Krämer, 2010; Wood, 2011). A putative model of how DynA might assist membrane fusion during cytokinesis is shown in Figure 4. DynA is accumulating at the site of septation, likely due to protein-protein contacts (MinJ, EzrA) and/or attraction by negatively charged phospholipids that are highly enriched at the septum. Owing to
DynA membrane binding in trans, the septal membrane is tethered, and likely curvature is induced, thereby leading to efficient fusion. This membrane fusion model is similar to the membrane fusion models for atlastin. Atlastin molecules on opposing membranes dimerize due to GTP binding (Moss et al., 2011). The induced conformational change stabilizes the atlastin oligomer and induces strong membrane curvature, which leads via a hemifusion state to full fusion (Moss et al., 2011).

In order to get more insights into the cellular roles of bacterial dynamins, screening experiments trying to identify physical or genetic partners are a valuable source. We have used a bacterial two-hybrid method (Karimova et al., 2005) to construct a B. subtilis whole genome library and screened for interacting proteins (Bürmann et al., 2012). Three candidate proteins were identified, RNaseY, YneK, and YwpG. RNaseY is a major RNA-degrading protein in Bacillus, involved in the degradation of messenger RNA and ribosomal RNA (Commichau et al., 2009; Segev et al., 2012). Interaction of RNaseY depends on the full-length DynA, which indicated that the membrane-associated DynA oligomer could be the target. Identification of DynA/ RNaseY interaction might indicate a role for bacterial dynamins other than supporting membrane fusion during cytokinesis. YneK and YwpG are both uncharacterized proteins. Interestingly, YneK is a putative transmembrane protein and interacts selectively with the membrane-binding domain D1 of DynA. Localization of YneK, YwpG, and RNaseY is similar compared to DynA showing membrane 
association and distinct foci (Bürmann et al., 2012). All three proteins are losing the ability to form foci in the absence of MinJ, which in turn was shown to be necessary for correct localization of DynA. This suggests that DynA plays a role in organizing subcellular localization of its binding partners.

\section{Conclusion}

We are just at the beginning to understand the molecular function of bacterial DLPs, and their biological role seems still an enigma. However, the broad conservation of these large GTPases in many bacterial genomes suggests an important role for the cell biology of bacteria. The close homology to DLPs of the mitofusin/Fzo class makes bacterial dynamins ideal tools to unravel the biochemical mechanism of membrane fusion. The bacterial dynamins are, like the bacterial actins a decade ago, a fascinating class of proteins that were long thought to be eukaryotic achievements. Thus, bacterial cell biology still offers many rewarding topics for future research.

Acknowledgements: I thank Prof. Reinhard Krämer (University of Cologne) for his continuous support and all members of my lab, in particular, Frank Bürmann, Nina Ebert, and Prachi Sawant (all from the University of Cologne) for their scientific contributions. I wish to thank Nina Ebert and Prachi Sawant for their help with the figure preparations and Gerrit Praefcke (University of Cologne) for the numerous stimulating discussions. The author thanks the anonymous referees for their valuable comments. Financial support from the Deutsche Forschungsgemeinschaft (SFB 635, project B8) is acknowledged.

Received April 23, 2012; accepted June 29, 2012

\section{References}

Alexander, C., Votruba, M., Pesch, U.E., Thiselton, D.L., Mayer, S., Moore, A., Rodriguez, M., Kellner, U., Leo-Kottler, B., Auburger, G., et al. (2000). OPA1, encoding a dynamin-related GTPase, is mutated in autosomal dominant optic atrophy linked to chromosome 3q28. Nat. Genet. 26, 211-215.

Anton, F., Fres, J.M., Schauss, A., Pinson, B., Praefcke, G.J., Langer, T., and Escobar-Henriques, M. (2011). Ugo1 and Mdm30 act sequentially during Fzo1-mediated mitochondrial outer membrane fusion. J. Cell Sci. 124, 1126-1135.

Bashkirov, P.V., Akimov, S.A., Evseev, A.I., Schmid, S.L., Zimmerberg, J., and Frolov, V.A. (2008) GTPase cycle of dynamin is coupled to membrane squeeze and release, leading to spontaneous fission. Cell 135, 1276-1286.

Bian, X., Klemm, R.W., Liu, T.Y., Zhang, M., Sun, S., Sui, X., Liu, X. Rapoport, T.A., and Hu, J. (2011). Structures of the atlastin GTPase provide insight into homotypic fusion of endoplasmic reticulum membranes. Proc. Natl. Acad. Sci. USA 108, 3976-3981.

Bramkamp, M., Emmins, R., Weston, L., Donovan, C., Daniel, R.A., and Errington, J. (2008). A novel component of the division-site selection system of Bacillus subtilis and a new mode of action for the division inhibitor MinCD. Mol. Microbiol. 70, 1556-1569.

Bürmann, F., Ebert, N., van Baarle, S., and Bramkamp, M. (2011). A bacterial dynamin-like protein mediating nucleotideindependent membrane fusion. Mol. Microbiol. 79, 1294-1304.

Bürmann, F., Sawant, P., and Bramkamp, M. (2012). Identification of interaction partners of the dynamin-like protein DynA from Bacillus subtilis. Commun. Integr. Biol. 5. (epub ahead of print, http://dx.doi.org/10.4161/cib.20215).

Byrnes, L.J. and Sondermann, H. (2011). Structural basis for the nucleotide-dependent dimerization of the large $\mathrm{G}$ protein atlastin-1/SPG3A. Proc. Natl. Acad. Sci. USA 108, 2216-2221.
Chappie, J.S., Acharya, S., Leonard, M., Schmid, S.L., and Dyda, F. (2010). G domain dimerization controls dynamin's assemblystimulated GTPase activity. Nature 465, 435-440.

Chappie, J.S., Mears, J.A., Fang, S., Leonard, M., Schmid, S.L., Milligan, R.A., Hinshaw, J.E., and Dyda, F. (2011). A pseudoatomic model of the dynamin polymer identifies a hydrolysis-dependent powerstroke. Cell 147, 209-222.

Chernomordik, L.V. and Kozlov, M.M. (2003). Protein-lipid interplay in fusion and fission of biological membranes. Annu. Rev. Biochem. 72, 175-207.

Cohen, M.M., Amiott, E.A., Day, A.R., Leboucher, G.P., Pryce, E.N. Glickman, M.H., McCaffery, J.M., Shaw, J.M., and Weissman, A.M. (2011). Sequential requirements for the GTPase domain of the mitofusin Fzo1 and the ubiquitin ligase SCFMdm30 in mitochondrial outer membrane fusion. J. Cell Sci. 124, 1403-1410.

Commichau, F.M., Rothe, F.M., Herzberg, C., Wagner, E., Hellwig, D., Lehnik-Habrink, M., Hammer, E., Volker, U., and Stulke, J. (2009). Novel activities of glycolytic enzymes in Bacillus subtilis: interactions with essential proteins involved in mRNA processing. Mol. Cell. Proteomics 8, 1350-1360.

Damke, H., Binns, D.D., Ueda, H., Schmid, S.L., and Baba, T. (2001). Dynamin GTPase domain mutants block endocytic vesicle formation at morphologically distinct stages. Mol. Biol. Cell 12, 2578-2589.

Daumke, O., Lundmark, R., Vallis, Y., Martens, S., Butler, P.J., and McMahon, H.T. (2007). Architectural and mechanistic insights into an EHD ATPase involved in membrane remodelling. Nature 449, 923-927.

Deatherage, B.L. and Cookson, B.T. (2012). Membrane vesicle release in Bacteria, Eukaryotes and Archaea: a conserved yet underappreciated aspect of microbial life. Infect. Immun. 80 , 1948-1957. 
Deatherage, B.L., Lara, J.C., Bergsbaken, T., Rassoulian Barrett, S.L., Lara, S., and Cookson, B.T. (2009). Biogenesis of bacterial membrane vesicles. Mol. Microbiol. 72, 1395-1407.

Delettre, C., Lenaers, G., Griffoin, J.M., Gigarel, N., Lorenzo, C., Belenguer, P., Pelloquin, L., Grosgeorge, J., Turc-Carel, C., Perret, E., et al. (2000). Nuclear gene OPA1, encoding a mitochondrial dynamin-related protein, is mutated in dominant optic atrophy. Nat. Genet. 26, 207-210.

DeVay, R.M., Dominguez-Ramirez, L., Lackner, L.L., Hoppins, S., Stahlberg, H., and Nunnari, J. (2009). Coassembly of Mgm1 isoforms requires cardiolipin and mediates mitochondrial inner membrane fusion. J. Cell Biol. 186, 793-803.

Downing, A.K., Driscoll, P.C., Gout, I., Salim, K., Zvelebil, M.J., and Waterfield, M.D. (1994). Three-dimensional solution structure of the pleckstrin homology domain from dynamin. Curr. Biol. 4, 884-891.

Faelber, K., Posor, Y., Gao, S., Held, M., Roske, Y., Schulze, D., Haucke, V., Noe, F., and Daumke, O. (2011). Crystal structure of nucleotide-free dynamin. Nature 477, 556-560.

Ferguson, S.M. and De Camilli, P. (2012). Dynamin, a membraneremodelling GTPase. Nat. Rev. Mol. Cell Biol. 13, 75-88.

Ford, M.G., Jenni, S., and Nunnari, J. (2011). The crystal structure of dynamin. Nature 477, 561-566.

Fres, J.M., Muller, S., and Praefcke, G.J. (2010). Purification of the CaaX-modified, dynamin-related large GTPase hGBP1 by coexpression with farnesyltransferase. J. Lipid Res. 51, 2454-2459.

Gao, H., Kadirjan-Kalbach, D., Froehlich, J.E., and Osteryoung, K.W. (2003). ARC5, a cytosolic dynamin-like protein from plants, is part of the chloroplast division machinery. Proc. Natl. Acad. Sci. USA 100, 4328-4333.

Gao, S., von der Malsburg, A., Dick, A., Faelber, K., Schroder, G.F., Haller, O., Kochs, G., and Daumke, O. (2011). Structure of myxovirus resistance protein a reveals intra- and intermolecular domain interactions required for the antiviral function. Immunity 35, 514-525.

Gao, S., von der Malsburg, A., Paeschke, S., Behlke, J., Haller, O., Kochs, G., and Daumke, O. (2010). Structural basis of oligomerization in the stalk region of dynamin-like MxA. Nature 465, 502-506.

Gasper, R., Meyer, S., Gotthardt, K., Sirajuddin, M., and Wittinghofer, A. (2009). It takes two to tango: regulation of $\mathrm{G}$ proteins by dimerization. Nat. Rev. Mol. Cell Biol. 10, 423-429.

Ghosh, A., Praefcke, G.J., Renault, L., Wittinghofer, A., and Herrmann, C. (2006). How guanylate-binding proteins achieve assembly-stimulated processive cleavage of GTP to GMP. Nature 440, 101-104.

Hales, K.G. and Fuller, M.T. (1997). Developmentally regulated mitochondrial fusion mediated by a conserved, novel, predicted GTPase. Cell 90, 121-129.

Hermann, G.J., Thatcher, J.W., Mills, J.P., Hales, K.G., Fuller, M.T., Nunnari, J., and Shaw, J.M. (1998). Mitochondrial fusion in yeast requires the transmembrane GTPase Fzo1p. J. Cell Biol. 143, 359-373.

Hu, J., Shibata, Y., Voss, C., Shemesh, T., Li, Z., Coughlin, M., Kozlov, M.M., Rapoport, T.A., and Prinz, W.A. (2008). Membrane proteins of the endoplasmic reticulum induce high-curvature tubules. Science 319, 1247-1250.

Hu, J., Shibata, Y., Zhu, P.P., Voss, C., Rismanchi, N., Prinz, W.A., Rapoport, T.A., and Blackstone, C. (2009). A class of dynamin-like GTPases involved in the generation of the tubular ER network. Cell 138, 549-561.

Ingerman, E., Perkins, E.M., Marino, M., Mears, J.A., McCaffery, J.M., Hinshaw, J.E., and Nunnari, J. (2005). Dnm1 forms spirals that are structurally tailored to fit mitochondria. J. Cell Biol. 170, 1021-1027.

Karimova, G., Dautin, N., and Ladant, D. (2005). Interaction network among Escherichia coli membrane proteins involved in cell division as revealed by bacterial two-hybrid analysis. J. Bacteriol. 187, 2233-2243.

Knott, A.B., Perkins, G., Schwarzenbacher, R., and Bossy-Wetzel, E. (2008). Mitochondrial fragmentation in neurodegeneration. Nat. Rev. Neurosci. 9, 505-518.

Koshiba, T., Detmer, S.A., Kaiser, J.T., Chen, H., McCaffery, J.M., and Chan, D.C. (2004). Structural basis of mitochondrial tethering by mitofusin complexes. Science 305, 858-862.

Kozlovsky, Y. and Kozlov, M.M. (2003). Membrane fission: model for intermediate structures. Biophys. J. 85, 85-96.

Krämer, R. (2010). Bacterial stimulus perception and signal transduction: response to osmotic stress. Chem. Rec. 10, 217-229.

Leipe, D.D., Wolf, Y.I., Koonin, E.V., and Aravind, L. (2002). Classification and evolution of P-loop GTPases and related ATPases. J. Mol. Biol. 317, 41-72.

Lemmon, M.A. (2007). Pleckstrin homology (PH) domains and phosphoinositides. Biochem. Soc. Symp. 81-93.

Low, H.H. and Löwe, J. (2006). A bacterial dynamin-like protein. Nature 444, 766-769.

Low, H.H. and Löwe, J. (2010). Dynamin architecture-from monomer to polymer. Curr. Opin. Struct. Biol. 20, 791-798.

Low, H.H., Sachse, C., Amos, L.A., and Löwe, J. (2009). Structure of a bacterial dynamin-like protein lipid tube provides a mechanism for assembly and membrane curving. Cell 139, 1342-1352.

Meeusen, S., DeVay, R., Block, J., Cassidy-Stone, A., Wayson, S., McCaffery, J.M., and Nunnari, J. (2006). Mitochondrial inner-membrane fusion and crista maintenance requires the dynamin-related GTPase Mgm1. Cell 127, 383-395.

Mettlen, M., Pucadyil, T., Ramachandran, R., and Schmid, S.L. (2009). Dissecting dynamin's role in clathrin-mediated endocytosis. Biochem. Soc. Trans. 37, 1022-1026.

Moss, T.J., Andreazza, C., Verma, A., Daga, A., and McNew, J.A. (2011). Membrane fusion by the GTPase atlastin requires a conserved C-terminal cytoplasmic tail and dimerization through the middle domain. Proc. Natl. Acad. Sci. USA 108, 11133-11138.

Niemann, H.H., Knetsch, M.L., Scherer, A., Manstein, D.J., and Kull, F.J. (2001). Crystal structure of a dynamin GTPase domain in both nucleotide-free and GDP-bound forms. EMBO J. 20, 5813-5821.

Obar, R.A., Collins, C.A., Hammarback, J.A., Shpetner, H.S., and Vallee, R.B. (1990). Molecular cloning of the microtubuleassociated mechanochemical enzyme dynamin reveals homology with a new family of GTP-binding proteins. Nature 347, 256-261.

Orso, G., Pendin, D., Liu, S., Tosetto, J., Moss, T.J., Faust, J.E., Micaroni, M., Egorova, A., Martinuzzi, A., McNew, J.A., et al. (2009). Homotypic fusion of ER membranes requires the dynamin-like GTPase atlastin. Nature 460, 978-983.

Pamp, S.J., Harrington, E.D., Quake, S.R., Relman, D.A., and Blainey, P.C. (2012). Single-cell sequencing provides clues about the 
host interactions of segmented filamentous bacteria (SFB). Genome Res. 22, 1107-1119.

Patrick, J.E. and Kearns, D.B. (2008). MinJ (YvjD) is a topological determinant of cell division in Bacillus subtilis. Mol. Microbiol. 70, 1166-1179.

Pawlowski, N. (2010). Dynamin self-assembly and the vesicle scission mechanism: how dynamin oligomers cleave the membrane neck of clathrin-coated pits during endocytosis. Bioessays 32, 1033-1039.

Praefcke, G.J. and McMahon, H.T. (2004). The dynamin superfamily: universal membrane tubulation and fission molecules? Nat. Rev. Mol. Cell Biol. 5, 133-147.

Praefcke, G.J., Kloep, S., Benscheid, U., Lilie, H., Prakash, B., and Herrmann, C. (2004). Identification of residues in the human guanylate-binding protein 1 critical for nucleotide binding and cooperative GTP hydrolysis. J. Mol. Biol. 344, 257-269.

Prakash, B., Praefcke, G.J., Renault, L., Wittinghofer, A., and Herrmann, C. (2000). Structure of human guanylate-binding protein 1 representing a unique class of GTP-binding proteins. Nature 403, 567-571.

Pucadyil, T.J. and Schmid, S.L. (2008). Real-time visualization of dynamin-catalyzed membrane fission and vesicle release. Cell 135, 1263-1275.

Ramachandran, R., Pucadyil, T.J., Liu, Y.W., Acharya, S., Leonard, M., Lukiyanchuk, V., and Schmid, S.L. (2009). Membrane insertion of the pleckstrin homology domain variable loop 1 is critical for dynamin-catalyzed vesicle scission. Mol. Biol. Cell 20, 4630-4639.

Santel, A. and Fuller, M.T. (2001). Control of mitochondrial morphology by a human mitofusin. J. Cell Sci. 114, 867-874.

Schmid, S.L. and Frolov, V.A. (2011). Dynamin: functional design of a membrane fission catalyst. Annu. Rev. Cell Dev. Biol. 27, 79-105.

Schwemmle, M. and Staeheli, P. (1994). The interferon-induced $67-k D a$ guanylate-binding protein (hGBP1) is a GTPase that converts GTP to GMP. J. Biol. Chem. 269, 11299-11305.

Segev, E., Smith, Y., and Ben-Yehuda, S. (2012). RNA dynamics in aging bacterial spores. Cell 148, 139-149.

Sesaki, H. and Jensen, R.E. (2001). UGO1 encodes an outer membrane protein required for mitochondrial fusion. J. Cell Biol. 152, 1123-1134.
Sesaki, H. and Jensen, R.E. (2004). Ugo1p links the Fzo1p and Mgm1p GTPases for mitochondrial fusion. J. Biol. Chem. 279, 28298-28303.

Shibata, Y., Voss, C., Rist, J.M., Hu, J., Rapoport, T.A., Prinz, W.A., and Voeltz, G.K. (2008). The reticulon and DP1/Yop1p proteins form immobile oligomers in the tubular endoplasmic reticulum. J. Biol. Chem. 283, 18892-18904.

Sievers, F., Wilm, A., Dineen, D., Gibson, T.J., Karplus, K., Li, W., Lopez, R., McWilliam, H., Remmert, M., Soding, J., et al. (2011). Fast, scalable generation of high-quality protein multiple sequence alignments using Clustal Omega. Mol. Syst. Biol. 7, 539.

Smirnova, E., Shurland, D.L., Ryazantsev, S.N., and van der Bliek, A.M. (1998). A human dynamin-related protein controls the distribution of mitochondria. J. Cell Biol. 143, 351-358.

van der Bliek, A.M. (1999). Functional diversity in the dynamin family. Trends Cell Biol. 9, 96-102.

van der Bliek, A.M. and Meyerowitz, E.M. (1991). Dynamin-like protein encoded by the Drosophila shibire gene associated with vesicular traffic. Nature 351, 411-414.

von der Malsburg, A., Abutbul-Ionita, I., Haller, O., Kochs, G., and Danino, D. (2011). Stalk domain of the dynamin-like MxA GTPase protein mediates membrane binding and liposome tubulation via the unstructured L4 loop. J. Biol. Chem. 286, 37858-37865.

Warnock, D.E. and Schmid, S.L. (1996). Dynamin GTPase, a forcegenerating molecular switch. Bioessays 18, 885-893.

Westermann, B. (2010). Mitochondrial fusion and fission in cell life and death. Nat. Rev. Mol. Cell Biol. 11, 872-884.

Wong, E.D., Wagner, J.A., Gorsich, S.W., McCaffery, J.M., Shaw, J.M. and Nunnari, J. (2000). The dynamin-related GTPase, Mgm1p, is an intermembrane space protein required for maintenance of fusion competent mitochondria. J. Cell Biol. 151, 341-352.

Wood, J.M. (2011). Bacterial osmoregulation: a paradigm for the study of cellular homeostasis. Annu. Rev. Microbiol. 65 , 215-238.

Zhang, Y., Gao, X., and Garavito, R.M. (2011). Biochemical characterization of human dynamin-like protein 1. J. Biochem. 150, 627-633. 